\title{
Mecanismos del comercio electrónico frente a la informalidad digital de las MIPYMES con emprendimientos verdes en Colombia y Nicaragua Mechanisms of electronic commerce in the face of digital informality of MSMEs with green enterprises in Colombia and Nicaragua \\ Liyei Mairena Misas Quiñonez' \\ lilleymairena0499@gmail.com \\ Código ORCID 0000-000I-6583-0695 \\ María José Silva Abarca ${ }^{2}$ \\ Código ORCID 0000-0003-4758-1271 \\ Ana Victoria Ruiz Centeno ${ }^{3}$ \\ Código ORCID 0000-0001-9109-3043
}

https://doi.org//0.5377/derecho.vli30.12225

Fecha de recibido: abril de 202I / Fecha de aprobación: agosto de 2021

Las MIPYMES representan el $\mathbf{9 9 . 6 \%}$ del total de las empresas en Colombia y casi el $\mathbf{9 0 \%}$ en Nicaragua, lo que demuestra su importancia para el sistema productivo de ambos países latinoamericanos. Sin embargo, dentro de esos porcentajes no se encuentran muchas MIPYMES con auténticas innovaciones ambientales, debido a que su entrada al mercado se caracteriza por ser retardado como consecuencia de la informalidad tanto presencial como digital, lo cual estanca el progreso de un desarrollo sostenible. Por tanto, se resulta necesario implementar herramientas o recursos que permitan el cumplimiento de los ODS (Objetivos de Desarrollo Sostenible) para hacer frente a la informalidad, así como utilizar tecnologías digitales, es decir, hacer uso del comercio electrónico para consolidar y fomentar proyectos verdes de micro, pequeñas y medianas empresas (MIPYMES), los cuales disminuyen los impactos negativos en los procesos de producción, distribución y consumo.

$$
\text { Palabras Clave }
$$

Comercio electrónico / MIPYMES / informalidad digital / sostenibilidad

\section{Abstract}

Small and medium-sized enterprises represent $99.6 \%$ of all companies in Colombia and almost $90 \%$ in Nicaragua, which demonstrates their importance for the production system of both Latin American countries. However, within these percentages there are not many SMEs with authentic environmental innovations, because their entry to the market is characterized by being delayed as a consequence of both face-to-face and digital informality, which stalls the progress of sustainable development. Therefore, it is necessary to implement tools or resources that allow compliance with the SDGs (Sustainable Development Goals) to deal with informality, as well as to use digital technologies, that is, to wield electronic commerce to consolidate and promote green projects of small and medium-sized enterprises (SMEs), which reduce the negative impacts on the production, distribution and consumption processes.

\section{Key words}

E-commerce, SMEs / digital informality / sustainability

\footnotetext{
${ }^{1}$ Estudiante de la Licenciatura en Derecho de la Universidad Cooperativa de Colombia y miembro del semillero Cundumi Dembele.

${ }^{2}$ Estudiante de la Licenciatura en Derecho y miembro de la Red Interdisciplinaria Iberoamericana de Investigadores e Investigadoras - Nodo Socio Jurídico.

${ }^{3}$ Estudiante de la Licenciatura en Derecho y miembro de la Red Interdisciplinaria Iberoamericana de Investigadores e Investigadoras - Nodo Socio Jurídico.
} 
Introducción. I. Comercio electrónico. I.I. Definición del comercio electrónico. I.2. Modalidades del comercio electrónico. I.3. Comercio electrónico en Nicaragua y Colombia. 1.4. Relación con emprendimientos verdes. 2. Metas de los ODS vinculados con los emprendimientos verdes. 3. MIPYMES en Nicaragua y Colombia. 3.I. Importancia de las MIPYMES. 4. Informalidad. 4.I. Herramientas o recursos frente a la informalidad que permiten el cumplimiento de los ODS. 5. Conclusiones.

\section{Introducción}

La cuarta revolución digital ha significado una transición para el ser humano que avanza sin precedentes, planteando cambios en nuestro estilo de vida, como ser más ecológicos. Estos cambios no significan una excepción para el ámbito comercial; al contrario, proponen una transformación en la industria, para aquellos que eligen adaptarse a este nuevo estilo de vida mediante la oferta de productos y servicios sostenibles, los cuales se ajustan tanto a los medios convencionales como a las nuevas tecnologías.

Esta transición ha implicado un reto para todos, sobre todo para las empresas a nivel mundial, ya que deben hacer mejoras en sus estándares para mitigar el impacto de sus operaciones, causando el nacimiento de los llamados emprendimientos verdes.

Junto a esta modificación se han presentado nuevos desafíos como la pandemia del COVID-19, que ha paralizado y cambiado nuestro mundo de una manera inimaginable. Estos cambios han impulsado el uso de herramientas digitales ante el choque económico que vivimos, siendo el comercio electrónico el más popular.

Sin embargo, debemos tener en cuenta la realidad de cada país y los diferentes sistemas económicos, por lo que el comercio electrónico se ha desarrollado de distintas formas alrededor del mundo y ha traído consigo un sin número de aspectos a considerar, como lo es la creciente presencia de la informalidad.

El presente trabajo busca responder cómo el comercio electrónico influye en la informalidad digital de las MIPYMES en Nicaragua y Colombia, enfatizando en los emprendimientos verdes; $y$, cuáles son las herramientas que pueden disminuir esta informalidad permitiendo el cumplimento de los Objetivos de Desarrollo Sostenible (de aquí en adelante denominado por sus siglas ODS). Esto se realizará por medio de una investigación teórica cualitativa.

\section{Comercio electrónico}

\section{I . I . Definición del comercio electrónico}

En materia jurídica no existe una definición del comercio electrónico acogida como única, por ello, varios doctrinarios y diversas organizaciones se han dedicado a otorgar sus propios significados.

\section{Revista de Derecho}


La Organización Mundial del Comercio (1998) indica que es la producción, distribución, comercialización, venta o entrega de bienes y servicios por medios electrónicos.

La Organización para la Cooperación y el Desarrollo Económicos (20l I) establece que es la compra o venta de bienes o servicios realizada en redes mediadas por computador, ya sea entre empresas, hogares, individuos, gobiernos y otras organizaciones públicas o privadas.

Vanhoose (2003) lo describe como cualquier proceso que conlleva el intercambio de propiedad o el uso de derechos para bienes y servicios vía electrónica vinculando dispositivos y comunicando interactivamente dentro de la red.

Aunque sean tres autores distintos, en todas las definiciones podemos distinguir dos elementos esenciales: el uso de la tecnología y el intercambio comercial, pues debe darse la compraventa o intercambio de bienes o servicios a través de medios electrónicos.

Por su parte, Nicaragua por el momento no ha creado una ley de comercio electrónico ni ha empezado iniciativa de ley al respecto, por lo que no se cuenta con una conceptualización prescrita en ley nacional. Empero, Colombia sí define qué es comercio electrónico en el inciso 2 del artículo 2 de la Ley 527 de 1999, estipulando que abarca las cuestiones suscitadas por toda relación de índole comercial, sea o no contractual, estructurada a partir de la utilización de uno o más mensajes de datos o de cualquier otro medio similar. Posteriormente, en el mismo artículo, indica que las relaciones de índole comercial comprenden, sin limitarse a ellas, las siguientes operaciones: toda operación comercial de suministro o intercambio de bienes o servicios; todo acuerdo de distribución; toda operación de representación o mandato comercial; todo tipo de operaciones financieras, bursátiles y de seguros; de construcción de obras; de consultoría; de ingeniería; de concesión de licencias; todo acuerdo de concesión o explotación de un servicio público; de empresa conjunta y otras formas de cooperación industrial o comercial; de transporte de mercancías o de pasajeros por vía aérea, marítima y férrea, o por carretera.

\section{I.2. Modalidades del comercio electrónico}

El uso masivo de las Tecnologías de la Información y la Comunicación ha propiciado que los usuarios de las mismas gocen de un amplio abanico de interacciones entre ellos y, como en la mayoría de los casos, los expertos facilitan clasificaciones.

En dependencia de los agentes económicos que intervienen en el comercio electrónico, se reconocen cuatro formas de intercambio comercial electrónico: el comercio electrónico de consumidor a empresas (C2B), el comercio electrónico de empresas a consumidor (B2C), el comercio electrónico de consumidor a consumidor (C2C) y el comercio electrónico de empresa a empresa (B2B).

Las dos más destacables son el comercio electrónico B2C y el comercio electrónico B2B. El primero consiste en que los oferentes o un operador digital, a través de una tienda electrónica, ofrecen a los potenciales clientes sus productos o servicios (Instituto de Investigaciones Jurídicas de la UNAM, 2018). El segundo, según Chrysovalantou Milliou y Lambros Pechlivanos (2008), se refiere a cualquier transacción comercial que 
se produce entre dos entidades separadas de negocio, a través de los mercados electrónicos basados en Internet, que benefician a las empresas participantes para reducir sus costos de transacción y mejorar la gestión de cadenas de suministro.

Esto último no debe confundirse con los negocios electrónicos, pues éstos son el uso de redes electrónicas para coordinar la toma de decisiones y la implementación de la producción de la organización, marketing y funciones de administración. (Vanhoose, 2003). Es decir, mientras el e-business está compuesto por todo el ciclo comercial (O’Connell, 2000), el comercio electrónico B2B sólo implica la transacción de negociación entre dos entidades comerciales.

\section{I.3. Comercio electrónico en Nicaragua y Colombia}

En Nicaragua, conforme a las estimaciones de Internet World Stats, en junio del 2020 había tres millones ciento veintiún mil usuarios de internet en Nicaragua. Lancaster (2020) señala en BuddeComm que el brote de coronavirus está teniendo un impacto significativo en las cadenas de producción y suministro a nivel mundial. Sin embargo, es improcedente pensar que esa cantidad de nicaragüenses realiza compras a través de internet, pues según Ortega (20l3), especialista nicaragüense en Marketing Digital y Comercio Electrónico, existe un rezago cuyas causas van desde la ausencia de una cultura digital hasta los requisitos, muchas veces imposibles de cumplir, que establece la banca para proporcionar la pasarela de pago que requieren los negocios para ofrecer el servicio de venta en línea.

Para remediar esta dilación y con el fin de que las MIPYMES puedan pasar de su página de Facebook al comercio electrónico, en conformidad con Ortega (2013), han surgido empresas que ofrecen la pasarela de pago y otros servicios que se requieran. En Nicaragua, una de ellas es Alfa, que desde el 20II ofrece toda la infraestructura tecnológica incluyendo la pasarela de pagos con CREDOMATIC y hospedaje, al igual que funcionalidades específicas como diseño adaptado a dispositivos móviles, aplicación en Facebook, estadísticas, entre otras.

Ahora, tal como se expresó en El Nuevo Diario en 2019, a pesar de la recesión económica que ha sufrido el país producto de la crisis sociopolítica, a partir del 2018 el e-commerce avanza a un mejor ritmo que hace cinco años y los usuarios están familiarizándose cada vez más con el sistema, lo cual se prueba con el hecho que en 2018 solamente un $2 \%$ de la población hacía compras y pagaba facturas de servicios en línea, pero en 2019 el $6.7 \%$ estaba haciendo pagos por internet, según datos publicados en un informe de la plataforma web HootSuite y la agencia creativa We Are Social. Esto se debe, explica Paladino (2019), a qué tiendas se han visto obligadas a cerrar físicamente por los costos y no tienen otra opción que continuar sus negocios de manera virtual, en vista que o lo hacen o no venden nada.

Respecto a la pandemia, Borgen (2020), director ejecutivo de la Cámara Nicaragüense de Internet y Telecomunicaciones (Canitel), expresa que ésta aceleró la transición hacia el comercio electrónico que se venía gestando y esta tendencia es irreversible. Esto se debe a que, además de mantener a flote algunos negocios y abastecer sin exponerse al contagio, el uso de la tecnología ha permitido que mediante el servicio de delivery y las aplicaciones de compra, como Hugo y Jumpers, los nicaragüenses adquieran comida, víveres, medicinas e incluso realicen cualquier tipo de mandado sin salir de casa. 
Por su parte, en Colombia, el comercio electrónico ha ido en aumento y el 2020, en especial, ha sido un año clave para acelerar el proceso por el distanciamiento social producto de la pandemia. El informe sobre la medición de indicadores de consumo del observatorio e-commerce del 2019, publicado en el 2020, indica que los principales puntos de acceso de bienes y servicios son el buscador y las redes sociales y que los medios de pago son la mayoría off-line, es decir compras en las que la transacción de pago es en efectivo, contra entrega o en punto de recaudo. Empero, esto cambió según el informe digital 2020, ya que los métodos de pago online más comunes fueron las tarjetas de créditos (57\%), seguidas por el dinero en efectivo (21\%), las transferencias bancarias (I7\%) y las billeteras electrónicas (5\%). Este cambio es producto de la prevención frente al contagio del COVID-19, además del aumento de los servicios y canales digitales.

Lo desarrollado demuestra que anteriormente no existía una cultura sobre la compra online; sin embargo, los medios electrónicos se convirtieron indiscutiblemente en el futuro del comercio, por lo cual, los entornos digitales están proliferando en Nicaragua y las MIPYMES están sacando adelante el comercio electrónico, pues están conscientes que les permite insertarse rápidamente en la oferta del mercado y resulta más barato que abrir una sucursal. De igual forma, en Colombia se evidencia un panorama positivo por la fuerte migración tanto de los consumidores como de los comerciantes, convirtiéndose en el país de América Latina en donde más ha crecido el e-commerce, según el informe de comportamiento del e-commerce en Colombia durante 2020 y perspectivas para 202 I elaborado por la Cámara de Comercio Colombiana de Comercio Electrónico.

\section{I.4. Relación con emprendimientos verdes}

A pesar de que el comercio electrónico está presente en todo tipo de emprendimientos, este trabajo se centrará en los negocios verdes, que se define como las actividades económicas en las que se ofertan bienes o servicios, que generan impactos ambientales positivos e incorporan buenas prácticas ambientales, sociales y económicas con enfoque de ciclo vida, contribuyendo a la conservación del ambiente como capital natural que soporta el desarrollo del territorio. (Ministerio de Ambiente, 2019)

La razón de este enfoque está fundamentada en que los emprendimientos verdes dan un valor agregado a la sociedad, en vista que generan ventajas de competitividad como: procesos más eficientes, menor uso tanto de materiales como de energía, reducción de residuos y un alto rendimiento de sus productos. Además, Colombia, según lo evidencia el informe Go Green: The Era of Sustainability, realizado por la consultora multinacional Nielseen, es el segundo país, después de India, en donde los consumidores demandan mayor sostenibilidad. Por su parte, en Nicaragua, desde hace algunos años han comenzado a surgir emprendimientos con fines de ayuda y protección al medio ambiente. Verbigracia, Mirna Castillo tuvo la iniciativa de reciclar productos para convertirlos en adornos, de manera que decidió emprender con su negocio CreoArte en el año 2008.

Por tanto, debido a las ventajas de competitividad y las crecientes demandas, así como necesidades ambientales, resulta evidente que en Colombia y Nicaragua el comercio 
electrónico debería ser sostenible y amigable con el planeta. Empero, estos emprendimientos aún no son numerosos y los existentes no tienen una gran visibilidad.

Por tanto, al tratarse de un sistema de mutualismo, por ayudarse uno al otro, a través del e-commerce se ha logrado una mayor perceptibilidad de los clientes, lo cual es justamente lo que se necesita que continúe sucediendo, pues muchas empresas tuvieron que adaptar su forma de venta a causa de la pandemia.

Asimismo, pese a que las medidas adoptadas para contener la propagación del COVID19 generaron impactos negativos en el precio de los productos primarios, en especial a los países que tienen una alta dependencia de los productos mineros y energéticos, el aislamiento también abrió una puerta de oportunidad de comenzar un negocio ecofriendly o llevar a cabo aquellos cambios en los emprendimientos, cuyo fin es mejorar los hábitos de consumo al mismo tiempo que se sale de la crisis económica que se presenta.

En Nicaragua, a causa del COVID-19, los nuevos restaurantes tuvieron que recurrir a crear cuentas en Facebook e Instagram y al servicio de delivery para poder vender y generar ingresos. Sin embargo, en vez de ocupar los típicos utensilios de desecho elaborados de plástico, decidieron comprar empaques, tenedores y cuchillos fabricados a base de bambú o que son biodegradables.

De igual forma, emprendimientos verdes creados antes de la pandemia optaron por el comercio electrónico. Por ejemplo, Paula Mangas y Diego Larios fundaron Ecokai en 2018 con el propósito de sustituir el uso del plástico con pajillas metálicas o de bambú e implementar bolsos a base de telas, poniendo en práctica la reutilización, y el día de hoy cuentan con su página en Instagram y Facebook.

En Colombia, Mercadolibre lanza en el 2019 la primera línea de productos sustentables para atender la demanda y desarrollar e impulsar emprendedores verdes, ya que en otras plataformas se divide en dos áreas: una relacionada con la venta de productos piratas y de contrabando a través de internet y la otra con bienes que, sin ser ilegales, se comercializan sin pagar ningún tipo de impuesto.

Por ende, los emprendimientos verdes son una necesidad actualmente, pues es obligación de todos buscar maneras de aminorar la contaminación e incentivar la compra y venta sostenible; $y$, el comercio electrónico es un excelente medio de publicidad, al igual que es un método para que estos negocios sigan a flote y susciten fuentes de empleo.

\section{Metas de los ODS vinculados con los emprendimientos verdes}

Como lo anunció el Programa de las Naciones Unidas para el Desarrollo (PNUD), los Objetivos de Desarrollo Sostenible, también conocidos como Objetivos Mundiales, se adoptaron por todos los Estados Miembros en 2015 como un llamado universal para poner fin a la pobreza, proteger el planeta y garantizar que todas las personas gocen de paz y prosperidad para 2030.

Se constituyen un total de 17 objetivos que se gestaron en la Conferencia de las Naciones Unidas sobre el Desarrollo Sostenible, celebrada en Río de Janeiro en 2012, los cuales están interrelacionados, lo que significa que el éxito de uno afecta el de otros, 
al igual que sucede con la efectividad de los emprendimientos verdes, lo que debería ser una prioridad para los países, incluidos Nicaragua y Colombia.

Esto se debe a que los emprendimientos verdes cumplen con una gran mayoría de los objetivos, por ejemplo: el número 8 "Trabajo decente y crecimiento económico", el número 9 "Industria, innovación e infraestructuras", el número II "Ciudades y comunidades comestibles", el número 12 "Producción y consumo responsable", el número I3 "Acción por el clima”, el número I4 "Vida submarina” y el número I5 "Vida de ecosistemas terrestres". Estos negocios ayudan a reducir la contaminación, conservar los recursos naturales, favorecer la integridad ambiental de los ecosistemas y estimular el uso sostenible de la biodiversidad responsable, todo como fuentes de la competitividad empresarial por medio del e-commerce, generando ingresos que facilitan la ejecución de los objetivos. Por ende, es toda una cadena de diversos beneficios interrelacionados que necesitan un fuerte apoyo en todas sus limitantes, como son de orden político y regulatorio, cultural, financiación, tecnológico y de infraestructura. El liderazgo empresarial es imprescindible para alcanzar los ODS; las empresas con una visión hacia al futuro, con la capacidad de sostenerse podrán construir y permanecer en la economía porvenir. "No es sólo por responsabilidad sino también por oportunidad, puesto que alcanzar los ODS reportará buenas oportunidades de negocio" (Pacto Mundial Red Española, 2019) en cuanto a reducción de costos, beneficios fiscales, o mejor acceso a financiación. Cumplir con las metas de los ODS es un punto clave para la supervivencia de las PYMES de aquí a unos años.

El Global Reporting Initiative es uno de los estándares mundiales de reportes de sostenibilidad más usados en el mundo, además de haber sido el primero a nivel mundial junto al financiamiento del Programa Suizo de Cooperación al Desarrollo Económico. En el caso de Colombia, se lanzó el programa de negocios competitivos para PYMES de GRI, que es una iniciativa que busca motivar a la PYMES a comprometerse con el desarrollo de reportes de sostenibilidad, de conformidad con el Pacto Global (2019), lo cual es un aspecto que brinda la posibilidad de generar valor de mediano y largo plazo, fortalecer la relación con los compradores que son cada vez más exigentes y generar conciencia colectiva de sostenibilidad.

\section{MIPYMES: Nicaragua y Colombia}

En cuanto a Nicaragua, la Ley de Promoción, Fomento y Desarrollo de la Micro, Pequeña y Mediana Empresa describe a las MIPYMES como todas aquellas micro, pequeñas y medianas empresas que operan como persona natural o jurídica, en los diversos sectores de la economía, siendo en general empresas manufactureras, industriales, agroindustriales, agrícolas, pecuarias, comerciales, de exportación, turísticas, artesanales y de servicios, entre otras.

Asimismo, esta Ley las clasifica según el número de trabajadores, los activos totales y las ventas anuales. Las micro empresas tienen de 5 a 10 trabajadores, sus activos totales son de hasta dos mil córdobas y sus ventas anuales son de hasta un millón de córdobas. Las pequeñas empresas tienen de 6 a 30 trabajadores, sus activos totales son de hasta un millón quinientos mil córdobas y sus ventas anuales son de hasta nueve millones de córdobas. Las medianas empresas tienen de 31 a 100 trabajadores, sus activos totales 
Mecanismos del comercio electrónico frente a la informalidad digital de las MIPYMES con emprendimientos verdes en Colombia y Nicaragua

son de hasta seis millones de córdobas y sus ventas anuales son de hasta cuarenta millones de córdobas.

Respecto a Colombia, las pequeñas y medianas empresas (PYMES) se definen en el Decreto 957 de junio del 2019 - reformando el artículo 2 de la Ley 540 - donde las clasifican por los ingresos de las actividades ordinarias anuales y al sector que pertenece. La nueva clasificación se explica mejor de la siguiente manera:

Tabla I

Clasificación de las PYMES en Colombia

\begin{tabular}{|l|l|l|l|}
\hline SECTOR & MICRO & PEQUEÑA & MEDIANA \\
\hline Manufacturero & $\begin{array}{l}\text { Inferior o igual a } \\
23.563 \text { UVT. }\end{array}$ & $\begin{array}{l}\text { Superior a 23.563 } \\
\text { UVT e inferior o } \\
\text { igual a 204.995 UVT. }\end{array}$ & $\begin{array}{l}\text { Superior } \\
204.195 \text { UVT e } \\
\text { inferior o igual a } \\
\text { I.736.565 UVT. }\end{array}$ \\
\hline Servicios & $\begin{array}{l}\text { Inferior o igual a } \\
32.988 \text { UVT. }\end{array}$ & $\begin{array}{l}\text { Superior a 32.988 } \\
\text { UVT e inferior o } \\
\text { igual a I3I.95I UVT. }\end{array}$ & $\begin{array}{l}\text { Superior } \\
\text { I3I.95I UVT e } \\
\text { inferior o igual a } \\
483.034\end{array}$ \\
\hline Comercio & $\begin{array}{l}\text { Inferior o igual a } \\
44.769 \text { UVT. }\end{array}$ & $\begin{array}{l}\text { Superior a 44.769 e } \\
\text { inferior o igual a } \\
43 I .196 \text { UVT. }\end{array}$ & $\begin{array}{l}\text { Superior } \\
431.196 \text { UVT e } \\
\text { inferior o igual a } \\
2.160 .692\end{array}$ \\
\hline
\end{tabular}

Aclaración: UVT representa Unidad de Valor Tributario.

Fuente: Elaboración propia en base a la regulación del Decreto 957 del 2019.

\section{I. Importancia de las MIPYMES}

El Banco Mundial (20I5) indica que, en Nicaragua, las MIPYMES, que representan casi el $90 \%$ del total de las compañías registradas, contribuyen al $40 \%$ de las exportaciones y al $35 \%$ del PIB, de acuerdo al CONIMIPYME; y, según el Banco Central de Nicaragua (20I8), su participación en la producción fue de $30.2 \%$ en el 2018.

En cuanto a la producción de empleos, el Banco Mundial (20I5) señala que generan alrededor de I,6 millones de puestos de trabajo, por lo que es lógico que, tal como expresan González Ruiz y Torres Treminio (2015), sean la mayor fuente generadora de empleos en el país, con el $72.88 \%$ del total de empleos a nivel nacional y con una mayor participación femenina del 54\%, concentrándose la mayor cantidad de empleos en los departamentos de Managua con 163,918 personas empleadas, Chinandega con 30,494, León 27,748 y Masaya con 22,725.

Paralelamente, para Colombia son de gran importancia porque son fuentes generadoras de empleo. Según cifras del Departamento Administrativo Nacional de Estadísticas, representan el $90 \%$ del sector productivo nacional y generan el $35 \%$ del PIB y el $80 \%$ del empleo de todo el país. No obstante, el $70 \%$ quiebran en los primeros cinco años por 
una organización básica e inexperta, por lo que tratan de sobrevivir por medio de la informalidad, "ya que no poseen la organización documental ni los requisitos básicos para lograr un músculo financiero y obtener ayuda de las entidades bancarias que les pueden aportar a su crecimiento", como expresa Cortés y Gómez (2019).

"Las PYMES son la clave para asegurar la prosperidad de los territorios, generar empleo, ofrecer oportunidades de formación y desarrollo de carreras profesionales y, por lo tanto, conseguir la erradicación de las desigualdades" (Pacto Mundial Red Española, 2019, p.13). Por ende, tienen una gran responsabilidad social donde se fundamentan en 4 pilares: los derechos humanos, las normas laborales, el medioambiente y la lucha contra la corrupción, todo esto para cumplir con un checklist de adquirir la licencia de operación. La sostenibilidad en una empresa representa un compromiso ambiental sin afectar la capacidad de las futuras generaciones en la creación o la satisfacción de sus propias necesidades. Es ser consciente del cambio climático, la pobreza y la dificultad en el futuro por la escasez del agua.

Los momentos de crisis fortalecen la resiliencia de un país. Las PYMES son máquinas generadoras de empleo y en especial para sectores vulnerables. En zonas rurales son la única fuente de empleo, pues son la "espina dorsal de la mayoría de las economías del mundo y desempeñan un papel fundamental en los países en desarrollo" (ONU, 2020) y es por su importancia que la Asamblea General decidió declarar el 27 de junio el día de las micro, pequeñas y medianas empresas, además de fomentar y concienciar acerca de su contribución con los ODS.

Sin embargo, uno de los problemas más fuertes que enfrentan es el cierre constante de los emprendimientos ante "procesos de escasez de recursos gerenciales y materiales y bajos niveles de planeación formalizada" (Vargas Martínez et al, 20I7), convirtiéndose en un gran generador de desempleo, "inhibiendo en la voluntad de nuevos empresarios" (Gómez O. \& Gómez R., 2018), quienes se ven en la obligación de encontrar otras alternativas de bajo costo.

\section{La informalidad}

Según la Organización Internacional del Trabajo (2007), la economía informal es el conjunto de actividades económicas que, tanto en la legislación como en la práctica, están tan poco contempladas por sistemas formales o no lo están en absoluto.

En cuanto a cifras, según Cepal (20I3), el 47\% del empleo informal en América Latina se trata de un fenómeno que presenta una elevada heterogeneidad y afecta más a ciertos colectivos del mercado laboral. Por ejemplo, $63 \%$ de los trabajadores del sector informal tienen sólo educación primaria y el $72 \%$ son pobres y forman parte del primer quintil de ingreso.

En lo referente a la informalidad medida desde las empresas, las cifras se encuentran en el 7I.I\%, debido a que, en su mayoría, los trabajadores informales laboran en microempresas (de I a 5 trabajadores) y el 75.7 \% lo hace en negocios que no llevan ningún tipo de registro contable. (Banco Central de Nicaragua, 2016) 
Respecto a Nicaragua, diversos estudios indican que presenta uno de los niveles más altos de informalidad en América Latina y, como es característico en los países en vía de desarrollo, las áreas rurales y las mujeres son las más afectadas por la informalidad.

De acuerdo con un artículo publicado por Romero (2020) en Vos Tv, la mitad de la población nicaragüense económicamente activa obtiene ingresos a través del empleo informal, principalmente en el sector servicio, agricultura y construcción.

En este sentido, el Banco Central de Nicaragua (2016), establece que el sector informal es el grupo de unidades de producción, que son empresas no constituidas de propiedad de jefes de hogares, incluidas las empresas informales por cuenta propia y las empresas de trabajadores informales.

Por tanto, para el Banco Central de Nicaragua, el empleo informal incluye:

$\rightarrow$ Trabajadores por cuenta propia (independientes sin empleados) en sus propias empresas del sector informal.

$\rightarrow$ Empleadores (independientes con empleados) en sus propias empresas del sector informal.

$\rightarrow$ Trabajadores familiares auxiliares, independientemente del tipo de empresa.

$\rightarrow$ Miembros de cooperativas de productores informales (no establecidas como entidades jurídicas).

$\rightarrow$ Trabajadores por cuenta propia que producen bienes exclusivos para el propio uso final de su hogar.

La Fundación Nicaragüense para el Desarrollo Económico y Social (2016), a través de la Encuesta de Empresas Sostenibles 2015, estimó que la informalidad empresarial total es del $88 \%$ y, en esa encuesta, una empresa informal es aquella que no lleva contabilidad o no tiene registro mercantil o no paga prestaciones sociales. Asimismo, la encuesta revela que el $\mathbf{8 9 . 8 \%}$ de las microempresas (de I a 5 trabajadores) eran informales.

Ahora, respecto a las causas de la informalidad, el economista nicaragüense Welbin Romero (2020) centra su teoría en que el problema de la informalidad se debe a que el Estado no promueve la generación de empleo formal entre sus políticas y la carga recae en las empresas privadas, las que anualmente generan entre 30 mil a 35 mil nuevas fuentes de empleos.

En la misma línea, Pérez Fajardo (2010) dice que los micro y pequeños empresarios, dedicados a la comercialización y la pequeña industria, consideran que la formalización es muy complicada o compleja y que el Estado lo que quiere es que tributen, pero no se preocupa por los beneficios que deberían obtener las MIPYMES. Sin embargo, continúa expresando el mismo autor, el Estado no es el único culpable, puesto que, por otra parte, la informalidad o la economía informal se debe a la poca o ninguna planeación de parte de las MIPYMES, que hacen negocios sin ninguna planificación o que su estrategia consiste en no tener ninguna.

En el caso de Colombia, la informalidad es a causa de la evasión de impuestos, referente a lo cual la Dirección de Impuestos y Aduanas Nacionales estipula que son 43 billones 
de pesos que se mueve anualmente, especialmente el no pago del IVA del 19\%, que representa el mayor inconveniente en materia de retrasos.

Ahora bien, el hincapié que se hace en la informalidad digital es que obstaculiza el recaudo del IVA y otros impuestos indirectos en operaciones B2C y B2B. Igualmente, hay plataformas digitales que no pagan impuestos e incluso venden productos que infringen la propiedad intelectual, por lo que son simplemente otra cara más del comercio ilegal.

Por tanto, se deben buscar mecanismos eficaces y normas expresas y claras que determinen cuándo y cómo se gravarán las transacciones a través de la red, debido a que, caso contrario, continuaría la poca estabilidad de los establecimientos y confianza de los compradores, conduciendo un retardo al consumo de estos productos, que presentan desafíos en cuanto a la producción de sus bienes y servicios y a su distribución y comercialización, lo cual es una limitante para países en vía de desarrollo como Colombia y Nicaragua, pues dificulta el correcto desenvolvimiento económico.

\section{I. Herramientas o recursos frente a la informalidad que permiten el cumplimiento de los ODS}

Ahora, una vez conocidas las causales de la informalidad, Pérez Fajardo (2010) plantea cuatro herramientas para darle solución a esta problemática. Primero propone la creación de políticas públicas coordinadas e integrales, las cuales deberán contener un conjunto de elementos como estabilidad macroeconómica, mejoras de la productividad tanto empresarial como estatal e instituciones que incentiven y faciliten la formalización empresarial y laboral. Seguidamente, contempla como necesario la realización de un diagnóstico integral del sector o sectores de las MIPYMES, el cual incluirá una evaluación detallada del impacto de todos los instrumentos de política pública y compatibilizada con las realidades nacionales. Posteriormente, sugiere la implementación de un Programa Integral de Desarrollo Empresarial enfocado a la formalización y fortalecimiento de capacidades empresariales de las MIPYME. Por último, pero probablemente la más importante, expone que se requiere de una actitud proactiva de parte de los empresarios que permita potenciar la productividad y el acceso sostenible a la formalización.

De igual manera, estimamos pertinente dos herramientas más. La primera se basa en los 6 ejes de acción propuestos por el BCN en 2016. Es decir, se debe trabajar en los problemas estructurales de la economía, en el desarrollo empresarial de las MIPYMES, en la simplificación de trámites para la formalización, en la articulación tanto productiva como profesional, en fortalecer la fiscalización del empleo y en medir la informalidad.

La segunda consiste en implementar un proceso de transición al régimen general basado en la experiencia del Régimen de Incorporación Fiscal que se complementa con un sistema intermedio de incorporación. Según Barreix y González (2020), se trata de "puente de plata" al régimen general por un periodo de transición donde se vaya pagando un porcentaje creciente de los tributos (IVA, Renta y CSS) generados. Asimismo, explican que, una vez que el contribuyente pase al régimen general, si se reducen sus ingresos, no podrá regresar al transitorio hasta transcurrido, por ejemplo, 3 a 4 años y los parámetros complementarios de control. 
Al poner en práctica estos recursos, al mismo tiempo que se reduce la informalidad, se cumple con los Objetivos de Desarrollo Sostenible. Primero que todo, lógicamente, entre más fáciles sean los trámites de formalización y el paso al régimen general, más personas, que tienen la voluntad de hacer el uso debido al medio ambiente y los recursos, van a emprender. De igual forma, es importante que se creen políticas públicas sostenibles y con sentido de innovación. Esto se debe a que el consumo y la producción continúan teniendo efectos destructivos sobre el planeta y la innovación y el progreso tecnológico son claves para descubrir soluciones duraderas para los desafíos económicos y medioambientales, como el aumento de la eficiencia energética y de recursos.

Respecto a Colombia, se han creado diferentes políticas públicas para el fomento de la formalización del empleo. El primer ejemplo es la Ley 2010 de 2019 que busca la promoción del empleo, la inversión, el fortalecimiento de las finanzas públicas, la progresividad, la equidad y la eficiencia del sistema tributario; al igual que conserva el objetivo principal de la Ley 1943 de 2018, que es combatir la informalidad simplificando los impuestos, lo que significa que se mantuvo el Impuesto Unificado bajo el Régimen Simple de Tributación que consiste en unificar la mayor cantidad de tributos y procedimientos, para así lograr el cumplimiento de la obligación tributaria por parte de las personas naturales y/o jurídicas que se acojan voluntariamente a este régimen de tributación.

La segunda política pública interpuesta es la iniciativa establecida en el acuerdo Distrital 780 de 2020, también conocido plan Marshall, que consiste en que el Distrito de Bogotá asume un porcentaje del costo del registro y renovación de la matrícula mercantil para los empresarios que se formalicen del 202I a 2026. Esto ocasiona que los empresarios se sientan apoyados por el Estado y tengan menos miedo por la inversión y posibilidad de pérdida, ya que disminuye el riesgo económico, por lo que se motivan a invertir en la formalización de sus emprendimientos.

Asimismo, a diferencia de otros países latinoamericanos, Colombia decidió regular, en el artículo 9l de la Ley 633 de 2000, los requisitos que deben seguir las páginas web y sitios de internet de origen colombiano para operar su actividad económica de carácter comercial en el internet, los cuales son: I) Inscribirse en el Registro Mercantil y 2) Suministrar a la Dirección y Aduanas Nacionales la información de transacciones económicas en los términos que esta entidad lo requiera. Además, hay un incentivo a cumplir con la regulación, porque de no hacerlo, las páginas web y sitios de internet de origen colombiano serán sometidas a cierre de su actividad y serán sujetos de sanciones, por ejemplo: una multa que impondrá la Superintendencia de Industria y Comercio. Esto implica que hay un reconocimiento de la existencia de los emprendimientos digitales y la exigencia de su formalización mediante la fuerza de coerción de las leyes nacionales y las consecuencias de su incumplimiento, por lo cual el e-commerce con enfoque verde accede al sistema financiero y al Plan Nacional de Negocios Verdes, que incluye diversos beneficios como posicionarnos en un lugar prioritario dentro de la agenda política, económica y social del país.

Por último, ante la dificultad de identificar las cuentas de redes sociales que hacen ventas en línea, la Secretaría de Hacienda de la Ciudad de Medellín implementó un software de analítica de datos que con insumos de diferentes fuentes de información y herramientas de búsqueda identificar los potenciales contribuyentes logrando encontrar 45 perfiles 
por hora cuando de manera manual por etiquetas se lograba identificar 5,8 perfiles (Centro de innovación y negocios, 2018), lo cual permitirá, tal como expresa Santo (2017), que el gobierno de Colombia ejecute retenciones en la fuente a las micro, pequeñas y medianas empresas desarrolladas en redes sociales con el fin de facilitar, acelerar y asegurar el recaudo del impuesto sobre la renta y sus complementarios; así como posibilitará que la Dirección de Impuestos y Aduanas Nacionales indique cada año quienes de las mencionadas empresas, de acuerdo con sus ingresos o patrimonio económico, deberán hacer la respectiva declaración de impuestos, para ejercer cierto control contra la evasión fiscal.

\section{Conclusiones}

Actualmente los consumidores son más estrictos en sus compras, puesto que exigen ciertos valores en los productos y servicios que adquieren, en especial revisan que sean amigables con el medio ambiente.

Para responder a este tipo de consumidor surgen los emprendimientos verdes, los cuales son innovaciones de micro, pequeñas y medianas empresas cuyas actividades económicas consisten en ofrecer bienes o servicios que impactan positivamente al ecosistema e incorporar buenas prácticas ambientales, sociales y económicas con un enfoque de ciclo vida, contribuyendo con los Objetivos de Desarrollo Sostenible vinculados con el comercio electrónico.

Por tanto, los objetivos de desarrollo sostenible que se ven cumplidos son: el número 8 "Trabajo decente y crecimiento económico", el número 9 "Industria, innovación e infraestructuras", el número II "Ciudades y comunidades sostenibles", el número 12 "Producción y consumo responsable", el número 13 "Acción por el clima", el número 14 "Vida submarina" y el número I5 "Vida de ecosistemas terrestres", debido a que todos ellos ayudan a reducir la contaminación, conservar los recursos naturales, favorecer la integridad ambiental de los ecosistemas y estimular el uso sostenible de la biodiversidad responsable, al mismo tiempo que funcionando como fuentes de la competitividad empresarial en el e-commerce.

No obstante, a pesar de los beneficios expuestos, al comercio electrónico con un sello sostenible le es difícil entrar al mercado y la carencia legal influye negativamente en la confiabilidad de los clientes en cuanto a la producción y comercialización, lo cual provoca que se estanque el progreso de estos emprendimientos y las mejoras consecuentes, porque más allá de que circulen es que lo hagan de una manera legal, contribuyendo al desarrollo del país y a la atenuación de los daños potenciales del medio ambiente.

Entonces, la formalización es un paso necesario para que las micro, pequeñas y medianas empresas se incorporen con todos sus derechos a la economía y puedan competir en igualdad de condiciones con otras empresas y ampliar su participación en el mercado.

Por tanto, es fundamental que se ejecuten los recursos propuestos para contrarrestar la informalidad presente en países latinoamericanos, en vista que son los que permitirán que haya un incremento económico individual y nacional como consecuencia de que el 
Estado llevará un mayor y mejor control de quienes deben pagar Impuesto sobre la Renta e Impuesto al Valor Agregado y de la cantidad correspondiente de cada uno.

Además, una vez dado este aumento vital para el desarrollo de Nicaragua, formalizadas las MIPYMES y seguidas las herramientas respecto al monotributo y el sistema intermedio de incorporación, los empresarios no sentirán que invierten más de lo que obtienen como remuneración de sus negocios, por lo que habrá más confianza en emprender cumpliendo todas las regulaciones, desde la creación e inscripción en los registros, lo cual incentivará a los Estados a crear políticas públicas que beneficien y ayuden a crecer a los empresarios.

Para el caso de Colombia a pesar que se ha realizado diferentes iniciativas y apoyo para la legalización aún persiste el desconocimiento frente a los beneficios de tal acto y las consecuencias de la informalidad, por ende se debe fortalecer redes de formalización a través de capacitaciones, y promover estrategias con seguimiento y control de estos.

Por último, se requiere un cambio de paradigma de una producción lineal hacia una producción circular para realizar una transformación positiva en el plano económico, social y ambiental. Además, el comercio, cuando se acompaña de la reglamentación apropiada, puede facilitar la transición hacia una economía verde al fomentar el intercambio de bienes y servicios ecológicos de una manera formal y digital, por lo cual las costumbres mercantiles y el derecho comercial se deben reevaluar con el objeto de adaptarse a las nuevas necesidades de hacer y de crear negocios. 


\section{Referencias bibliográficas}

Asamblea Nacional (2008). Ley 645: Ley de Promoción de Fomento y Desarrollo de la Micro, Pequeña y Mediana Empresa. Publicada en La Gaceta Diario Oficial No. 28, del 24 de enero. Managua, Nicaragua.

Baca Castellón, L. (2017). Nicaragua gatea en comercio electrónico. Recuperado de: https://www.laprensa.com.ni/2017/02/09/economia/2 179760-nicaragua-gateacomercio-electronico

Banco Central de Nicaragua. (2016) Determinantes para la informalidad en Nicaragua. Recuperado de: https://www.bcn.gob.ni/estadisticas/revista/trabajos_vollll/Brenes_y_Cruz_2016 .pdf

Banco Central de Nicaragua. (2018). Informe Anual 2018. Recuperado de: https://www.ben.gob.ni/publicaciones/periodicidad/anual/informe_anual/20I8/Inf orme\%20Anual\%202018.pdf

Banco Mundial. (2015). Por qué para Nicaragua es vital apoyar a las micro, pequeñas y medianas empresas. Recuperado de: https://www.bancomundial.org/es/news/feature/2015/03/20/nicaragua-micropequenas-medianas-empresas

Barreix \& González. (2020). Tributación de las MIPYMES: un régimen para el $80 \%$ de los contribuyentes que pagan el $2 \%$. Recuperado de: https://blogs.iadb.org/gestionfiscal/es/tributacion-para-mipymes-post-pandemia/

Cámara Colombiana de Comercio Electrónico. (2020). Informe comportamientos del ecommerce en Colombia durante 2020 y perspectivas para el 2021 . Recuperado de: https://www.ccce.org.co/wp-content/uploads/2020/10/informecomportamiento-y-perspectiva-ecommerce-2020-202I.pdf

Centro de Innovación y negocios. (2018). Disminuir la evasión de impuestos asociada a las ventas por internet: el reto de la secretaría de hacienda. Recuperado de: https://www.rutanmedellin.org/es/programas-vigentes/2-uncategorised/594reto-de-hacienda

Chen, Marta (2012). La economía informal: definiciones, teorías y políticas. Documento de Trabajo de WIEGO No. I.

Concejo de Bogotá D.C.(2020).Acuerdo 780 de 2020:Por el cual se establecen incentivos para la reactivación económica, respecto de los impuestos predial unificado e industria y comercio, producto de la situación epidemiológica causada por el Coronavirus (Covid 19), se adopta el impuesto unificado bajo el régimen simple de tributación (simple) en el Distrito Capital, se fijan las tarifas consolidadas del mismo, se establecen 
Mecanismos del comercio electrónico frente a la informalidad digital de las MIPYMES con emprendimientos verdes en Colombia y Nicaragua

beneficios para la formalización empresarial y se dictan otras medidas en materia tributaria $y$ de procedimiento. Recuperado de: https://www.shd.gov.co/shd/sites/default/files/files/impuestos/Acuerdo-780-de2020.pdf

Congreso de la República de Colombia. (1999). Ley 527 de 1999: Por medio de la cual se define y reglamenta el acceso y uso de los mensajes de datos, del comercio electrónico y de las firmas digitales, y se establecen las entidades de certificación y se dictan otras disposiciones.

Recuperado

de:

https://www.alcaldiabogota.gov.co/sisjur/normas/Norma I.jsp?i=4276

Congreso de la República de Colombia.(2000).Ley 633 de 2000:Por la cual se expiden normas en materia tributaria, se dictan disposiciones sobre el tratamiento a los fondos obligatorios para la vivienda de interés social y se introducen normas para fortalecer las finanzas de la Rama Judicial. Recuperado de: https://www.funcionpublica.gov.co/eva/gestornormativo/norma.php?i=6285

Congreso de la República de Colombia. (2018). Ley 1943 de 20 I8: Por la cual se expiden normas de financiamiento para el restablecimiento del equilibrio del presupuesto general $y$ se dictan otras disposiciones. Recuperado de: http://www.secretariasenado.gov.co/senado/basedoc/ley_1943_20I8.html

Congreso de la República de Colombia.(2019).Ley 2010 de 2019:por medio de la cual se adoptan normas para la promoción del crecimiento económico, el empleo, la inversión, el fortalecimiento de las finanzas públicas y la progresividad, equidad y eficiencia del sistema tributario. Recuperado de: http://www.secretariasenado.gov.co/senado/basedoc/ley_2010_2019.html

Consejo General de la Organización Mundial de Comercio. (1998). "Work Programme on Electronic Commerce: Adopted by the General Council on 25 September 1998”.

Cortés Osmar \& Gómez Adriana. (2019). La informalidad ligada a la competitividad de las Pymes en Colombia. Recuperado de: https://www.aspromer.com/wpcontent/uploads/2019/07/PONENCIA-FINAL_ADRIANA-DEL-PILAR.pdf

De Soto, Hernando (1987). El otro sendero: La revolución invisible en el tercer mundo. México, D. F.

El Nuevo Diario. (2017). CreoArte: El proyecto verde de una emprendedora. Recuperado de: https://www.elnuevodiario.com.ni/economia/4I5I8I-creoarte-proyectoverde-emprendedora/

FUNIDES (2016). Coyuntura Económica Nicaragua: Segundo Informe 2016. Managua, Nicaragua.

González Ruiz, K. \& Torres Treminio, M. (20I5). Contribución de las Micro, Pequeñas y Medianas Empresas (MIPYMES) urbanas a la economía de Nicaragua, 2014. Universidad Nacional Agraria, UNA. 
International World State. (2020). Mexico and Central America Telecommunications Report. Recuperado de: https://www.internetworldstats.com/central.htm\#ni

Instituto de Investigaciones Jurídicas de la UNAM. (2018). El Comercio Electrónico y Principios Económicos-Comerciales. Universidad Nacional Autónoma de México. Recuperado de: https://archivos.juridicas.unam.mx/www/bjv/libros// 0/4667/4.pdf

La Voz del Sandinismo. (2019). Movimiento Guardabarranco capacita a jóvenes emprendedores en Managua. Recuperado de: https://www.lavozdelsandinismo.com/nicaragua/2019-03-0I/movimientoguardabarranco-capacita-a-jovenes-emprendedores-en-managua/

Milliou, Chrysovalantou and Pechlivanos. (2008). Lambros: "Choosing Between Different Forms of b2b Electronic Marketplaces". Greece, Athens University of Economics and Business- Department of International and European Economics Studies.

Ministerio de Ambiente. (2019). ¿Qué son los negocios verdes? Gobierno colombiano. Recuperado de: https://www.minambiente.gov.co/index.php/component/content/article/I38I plantilla-negocios-verdes-y-sostenibles-38

Ministerio de Industria y Comercio Colombiano (2019). Decreto 957 de 2019: Por el cual se adiciona el capítulo 13 al Título I de la Parte 2 del Libro 2 del Decreto 1074 de 2015, Decreto Único del Sector Comercio, Industria y Turismo y se reglamenta el artículo $2^{\circ}$ de la Ley 590 de 2000, modificado por el artículo 43 de la Ley 1450 de 2011 Recuperado de: https://www.mincit.gov.co/normatividad/decretos/2019/decreto-957-por-elcual-se-adiciona-el-capitulo- 13

Nielsen. (2019). Go Green: The Era of Sustainability. Recuperado de: https://www.nielsen.com/wp-content/uploads/sites/3/2019/06/report-latamgoing-green.pdf

Organización de las Naciones Unidas (2020). Día de las microempresas y las pequeñas y medianas empresas 27 de junio. Recuperado de: https://www.un.org/es/observances/micro-small-medium-businesses-day

O'Connell, B. (2000). Business to Business: Las Claves para Rentabilizar el Comercio Electrónico. Esther Gil. España.

Organización Internacional del Trabajo. (2002). El trabajo decente y la economía informal. 90a Reunión 2002. Informe VI. Ginebra.

OIT (1972). Employment, incomes and equality: A strategy for increasing productive employment in Kenya. Ginebra.

Organization for Economic Co-operation and Development. (201 I). Guide to Measuring the Information Society. pág. 74. Recuperado en: https://www.oecdilibrary.org/science-and-technology/ 
Mecanismos del comercio electrónico frente a la informalidad digital de las MIPYMES con emprendimientos verdes en Colombia y Nicaragua

Ortega Ulloa, J. (2013). Comercio electrónico en Nicaragua: Hablemos de plataformas ecommerce y el caso ALFA. juanortega.ni. Recuperado de: https://medium.com/juanortega-ni/comercio-electrónico-en-nicaraguahablemos-de-plataformas-e-commerce-y-el-caso-alfa-c6afd4fdaf6b

Pacto global red Colombia (2019). GRI, sostenibilidad desde las PYMES. Recuperado de: https://www.pactoglobal-colombia.org/news/gri-la-sostenibilidad-desde-laspymes.html

Pacto mundial Red española. (2019). Guías para PYMES ante los objetivos de desarrollo sostenible. Recuperado de: https:/www.cepyme.es/wpcontent/uploads/2019/I I/Gu\%C3\%ADa-para-pymes-ante-los-ODS.pdf

Portafolio.co. (2019). Emprendimientos verdes conquistaron al mercado Colombiano. Recuperado de: https://www.portafolio.co/negocios/emprendimiento

Programa de las Naciones Unidas. (2015) ¿Cuáles son los objetivos de desarrollo sostenible? Recuperado de: https://www.undp.org/content/undp/es/home/sustainabledevelopment-goals.html

Romero, J. (2020) Empleo informal continúa creciendo en Nicaragua. Vos TV. Managua. Recuperado de: https://www.vostv.com.ni/economia/I273 I

Santo, J. (2017). Legislación vigente en materia tributaria del comercio electrónico (ecommerce) en Colombia y la necesidad de un pronunciamiento por parte del legislador. Revista Academia \& Derecho, Año 8, N 15, 2017, pp. 85-II0. Recuperado de: https://revistas.unilibre.edu.co/index.php/academia/article/download/4332/3679/ 7293

Van Hoose. D. (2003). e.commerce electronics. Ohio, Baylor University, South Western Thomson Learning.

Vidaurre, A. (2019). La crisis empuja el comercio digital en Nicaragua. Recuperado de: https://www.elnuevodiario.com.ni/economia/496894-crisis-nicaragua-comercioelectronico/

Westfield Business School. (20I0). Las MIPYMES y la Economía Informal en Nicaragua. Recuperado de: https://blog.i-ead.com/2010/06/07/las-mipymes-y-la-economiainformal-en-nicaragua/ 